\title{
Expression of immune check point gene TIM-3 in patients newly diagnosed with acute myeloid leukemia: Significance and impact on outcome
}

\author{
AMANY M. KAMAL ${ }^{1}$, NERMEEN A. NABIH ${ }^{2}$, NOORAN S. ELLEBOUDY ${ }^{3}$ and SARA M. RADWAN ${ }^{1}$ \\ ${ }^{1}$ Department of Biochemistry, Faculty of Pharmacy, Ain Shams University, Cairo 11566; ${ }^{2}$ Department of Internal Medicine, \\ Clinical Hematology and Bone Marrow Transplantation Unit, Faculty of Medicine, Ain Shams University, Cairo 11591; \\ ${ }^{3}$ Department of Microbiology and Immunology, Faculty of Pharmacy, Ain Shams University, Cairo 11566, Egypt
}

Received October 9, 2020; Accepted January 12, 2021

DOI: $10.3892 / \mathrm{ol} .2021 .12587$

\begin{abstract}
Despite recent advancements in the therapeutic landscape of acute myeloid leukemia (AML), the prognosis of patients remains poor. Immune check point inhibitors have been investigated in hematological malignancies, including AML; however, the role of T-cell immunoglobulin and mucin domain 3 (TIM-3) in AML has not yet been fully elucidated. Thus, the present study aimed to investigate TIM-3 gene expression in patients with AML and determine its associations with prognostic variables and clinical outcome. A total of 60 patients newly diagnosed with AML and 15 healthy matching individuals were recruited in the present study, and reverse transcription-quantitative PCR analysis was performed to detect TIM-3 expression. The results demonstrated that TIM-3 expression was significantly upregulated in patients with AML compared with that in healthy individuals $(\mathrm{P}<0.001)$. In addition, patients with extramedullary disease (EMD) exhibited significantly lower median TIM-3 expression levels compared with those without EMD $(\mathrm{P}=0.001)$. Furthermore, patients with high TIM-3 expression had significantly lower complete remission rates following induction chemotherapy compared with those with low TIM-3 expression $(\mathrm{P}=0.004)$. High TIM-3 expression was significantly associated with lower overall survival rates during the 1 -year follow-up $(\mathrm{P}=0.001)$. Taken together, the results of the present study suggest that TIM-3 may act as a biomarker of a poor prognosis in patients with AML, and be used as a therapeutic target.
\end{abstract}

Correspondence to: Dr Nermeen A. Nabih, Department of Internal Medicine, Clinical Hematology and Bone Marrow Transplantation Unit, Faculty of Medicine, Ain Shams University, 38 Ramsis Street, Cairo 11591, Egypt

E-mail: nermeen.adel@med.asu.edu.eg

Key words: gene expression, T lymphocytes, T-cell immunoglobulin and mucin domain 3 , reverse transcription-quantitative PCR, acute myeloid leukemia, induction chemotherapy, prognosis

\section{Introduction}

Acute myeloid leukemia (AML) is an aggressive highly heterogeneous hematological malignancy associated with a poor prognosis and high mortality rate (1). According to the SEER cancer statistics of 2018, the age-adjusted incidence of AML was 4.3 per 100,000 annually in the United States while the mortality rate was 2.8 per 100,000 with an estimated median overall survival (OS) time of AML of 8.5 months and 2-year and 5-year OS rates were 32.0 and $24.0 \%$, respectively (2). There is a high incidence of extramedullary disease (EMD) in AML at the initial diagnosis, during treatment and at relapse. Patients with AML and EMD have a worse prognosis than those without EMD (3). Conventional chemotherapy and hematopoietic stem cell transplantation remain the standard choice of care for patients with AML (4). However, resistance and poor tolerance to chemotherapy, treatment-related mortality and the high relapse rate create an urgent need to develop more effective and tolerable novel therapies for patients with AML, such as immune-based therapies $(4,5)$.

Inhibitory checkpoints are part of the normal immune system that function to turn off an immune response. These molecules interrupt T-cell activation and proliferation, and decrease cytokine production, which is mandatory for the establishment of peripheral tolerance during normal immune responses (6). Research on the immune microenvironment of AML has revealed that leukemia cells manipulate the immune system by a dynamic process called immunoediting, through which it takes advantage of the normal inhibitory checkpoints by expressing the ligands of these checkpoint receptors, thus resulting in T-cell exhaustion, a process of gradual loss of T-cell function and downregulation of the immune system. This concept may potentially explain immune escape by both solid and hematological malignancies $(6,7)$.

Exhausted $\mathrm{T}$ cells are characterized by increased expression of several inhibitory receptors, such as programmed cell death protein-1, T-cell immunoglobulin mucin domain 3 (TIM-3) and lymphocyte activation gene-3 (8). TIM-3 is a negative regulatory receptor expressed on $\mathrm{CD}^{+}$and $\mathrm{CD}^{+}$ $\mathrm{T}$ cells, T-regulatory cells and dendritic cells; it plays a key role in inhibiting Th1 responses and the expression of cytokines, 
such as tumor necrosis factor and $\gamma$-interferon $(8,9)$. There are four binding ligands that have been identified for TIM-3, including carcinoembryonic antigen cell adhesion molecule 1, high-mobility group protein B1, phosphatidylserine and galectin-9 (Gal-9) (9).

Several studies have demonstrated a close association between TIM-3 expression and tumor-associated immune suppression (10,11). TIM-3 and its ligand, Gal-9, are expressed on AML blast and leukemic stem cells (12). In addition, there is a reported increase in the percentage of TIM-3-expressing $\mathrm{CD}^{+} \mathrm{T}$ cells circulating in the blood of patients with AML compared with that in healthy individuals (13). However, further studies are required to confirm these results. The present study aimed to investigate TIM-3 expression in patients newly diagnosed with AML, as well as to determine its association with different prognostic variables and further investigate the impact of TIM-3 expression status on the clinical outcome.

\section{Patients and methods}

Patients. A total of 60 patients newly diagnosed with AML were recruited from the Department of Internal Medicine, Clinical Hematology and Bone Marrow Transplantation Unit, Ain-shams University Hospitals (Cairo, Egypt) between January 2018 and December 2018. All patients were treated and followed up for 12 months. A total of 15 healthy, age and sex matched individuals were enrolled to serve as controls. The patients included 24 men and 36 women, age range, 20-68 years, mean age of $53.4 \pm 12.9$ years, while the healthy controls included 7 men and 8 women, with age range, 35-56 years, mean age of $46.9 \pm 6.3$ years. The diagnosis was made according to the criteria of the French-American-British (FAB) classification and World Health Organization (14). The diagnosis was based on the morphological findings from Wright-Giemsa-stained smears of bone marrow aspirates, combined with immunophenotyping analyses of leukemic cells using diagnostic kits [CD34, CD13, CD33, myeloperoxidase, human leukocyte antigen-DR isotype (HLA-DR) HLA-DR, CD117, CD2 and CD19; Beckman Coulter, Inc.] (15) and cytogenetic studies by fluorescence in situ hybridization (FISH) using a locus-specific identifier DNA probe (fluorophore-labeled; Abbott Molecular). Dual-color FISH and visualization of hybridization signals by fluorescence microscopy were performed to detect the presence of recurrent cytogenetic abnormalities, as previously described by Fröhling et al (16).

A total of three cytogenetic risk groups were distinguished in AML, and patients with $\mathrm{t}(15 ; 17), \mathrm{t}(8 ; 21)$ and inv(16) were assigned to the 'favorable risk' group, while patients with $(3 q), \operatorname{del}(5 q),-5 /-7$ or complex karyotype were assigned to the 'poor risk' group. The remaining patients, including normal karyotype, were assigned to the 'intermediate risk' group, and patients with intermediate or poor risk cytogenetics had an unfavorable prognosis (17).

The present study was approved by the Ethical Committee of Research, Faculty of Medicine, Ain Shams University, and performed in accordance with the Declaration of Helsinki. Written informed consent was provided by all participants prior to the study start. The inclusion criteria for the patients were: i) Age $>18$ years; and ii) newly diagnosed AML and fit to receive chemotherapy. The exclusion criteria for the patients were: i) Secondary AML; ii) relapsed AML; and iii) unfit to receive chemotherapy. The included patients provided a detailed history, subjected to a clinical examination, and had other data extracted and recorded from the patient files. All patients received induction standard chemotherapy in accordance with NCCN 2019 guidelines for AML (PETHEMA protocol for APL M3 and 3+7 protocol for other types of AML) (17). By the end of the first induction, all surviving patients were assessed regarding their responsiveness to chemotherapy. Assessment included a full blood work up, as well as a bone marrow (BM) examination. Patients were classified into responders, who attained complete remission (CR), and non-responders, who were refractory to chemotherapy. CR was defined as an absolute neutrophilic count $>1,000 / \mu 1$, a platelet count $\geq 100,000 / \mu 1$ and $<5 \%$ BM blasts in a normocellular marrow, with no evidence of EMD. Patients who had achieved CR following induction chemotherapy were subjected to consolidation with either allogeneic stem cell transplantation, if they had poor risk cytogenetics and a matched sibling donor, or in cases where donors were unavailable and/or patients had a good risk from the start, high-dose chemotherapy (consolidation phase of PETHEMA protocol for APL M3 and high-dose cytosine arabinoside for other types of AML) (18). Overall survival time was defined as the time from the date of diagnosis until mortality. Patients still alive were censored at the end of the 12 months as only 1-year survival was assessed in the current study.

Reverse transcription-quantitative $(R T-q) P C R$. Peripheral blood samples were withdrawn from controls and from patients with AML prior to treatment, and contained in vacutainer tubes containing $\mathrm{Na}_{2}$ EDTA to determine the complete blood count and for total RNA purification from human whole blood. Total RNA was extracted using extraction buffer (present in the kit) and purified from human whole blood using the QIAamp RNA blood mini kit (Qiagen $\mathrm{GmbH}$ ), according to the manufacturer's protocol. Total RNA was reverse transcribed into cDNA using the High Capacity cDNA Reverse Transcription kit (Applied Biosystems; Thermo Fisher Scientific, Inc.), according to the manufacturer's protocol, and stored at $-20^{\circ} \mathrm{C}$ until subsequent experimentation. qPCR was subsequently performed using the Rotor-Gene $\mathrm{Q}^{\circledR}$ Real-Time PCR cycler (Qiagen $\mathrm{GmbH}$ ), with standard thermocycling conditions according to the Thermo Fisher Scientific Inc. protocol $\left(95^{\circ} \mathrm{C}\right.$ for $10 \mathrm{~min}$, followed by 40 cycles of $95^{\circ} \mathrm{C}$ for $15 \mathrm{sec}$ and $60^{\circ} \mathrm{C}$ for $1 \mathrm{~min}$ ) and the Taqman assay specific for TIM-3 (catalog no. Hs00958618_m1; Thermo Fisher Scientific, Inc.). Relative expression levels were normalized to the internal reference gene GAPDH (catalog no. Hs02786624_g1). The primer sequences used were as follows: TIM-3 forward, 5'-CCAAAT CCCAGGCATAAT-3' and reverse, 5'-AAGCGACAACCC AAAGGT-3', and GAPDH forward, 5'-GTCTCCTCTGAC TTCAACAGCG-3' and reverse, 5'-ACCACCCTGTTGCTG TAGCCAA-3'. The expression levels in unknown samples were normalized and analyzed by the $2^{-\Delta \Delta \mathrm{Cq}}$ method where $\Delta \Delta \mathrm{Cq}=\left(\mathrm{Cq}_{\text {target gene }}-\mathrm{Cq}_{\mathrm{GAPDH}}\right)$ sample- $\left(\mathrm{Cq}_{\text {target gene }}-\mathrm{Cq}_{\mathrm{GAPDH}}\right)$ calibrator (19).

Statistical analysis. Statistical analysis was performed using SPSS 23.0 software (IBM Corp.) and MedCalc 18.2.1 
Table I. Demographic data and clinical characteristics of the studied groups.

\begin{tabular}{|c|c|c|}
\hline Characteristic & Control group & AML group \\
\hline \multicolumn{3}{|l|}{ Sex, n $(\%)$} \\
\hline Male & $7(46.7)$ & $24(40.0)$ \\
\hline Female & $8(53.3)$ & $36(60.0)$ \\
\hline Age, years ${ }^{\mathrm{b}}$ & $46.9 \pm 6.3$ & $53.4 \pm 12.9$ \\
\hline Hemoglobin, g/dl & $14.5(13.9-15.6)$ & $7.9(5.9-9.6)$ \\
\hline WBCs, $1 \times 10^{3}$ cells $/ \mu 1^{\mathrm{a}}$ & $5.1(4.1-7.8)$ & $17.6(3.2-43.2)$ \\
\hline Absolute neutrophil count, $1 \times 103$ cells $/ \mu 1^{\mathrm{a}}$ & $4.2(3.6-5.9)$ & $1.9(1-7.6)$ \\
\hline Absolute lymphocytic count, $1 \times 10^{3}$ cells $/ \mu 1^{\mathrm{a}}$ & $2.3(1.9-2.6)$ & $1.6(1-12.7)$ \\
\hline PB blast count $(\%)^{\mathrm{a}}$ & - & $34(10-62)$ \\
\hline BM blast count $(\%)^{\mathrm{a}}$ & - & $36(25-71)$ \\
\hline TIM-3 fold expression ${ }^{a}$ & $1.07(0.84-1.12)$ & $2.99(1.45-5.65)$ \\
\hline \multicolumn{3}{|l|}{ Extramedullary disease, $\mathrm{n}(\%)$} \\
\hline+ & - & $8(13.3)$ \\
\hline- & - & $52(86.7)$ \\
\hline FAB classification, n $(\%)$ & - & \\
\hline M0 & - & $8(13.3)$ \\
\hline M1 & - & $4(6.7)$ \\
\hline M2 & - & $12(20.0)$ \\
\hline M3 & - & $12(20.0)$ \\
\hline M4 & - & $24(40.0)$ \\
\hline Prognosis according to cytogenetic studies, $\mathrm{n}(\%)$ & - & \\
\hline Favorable & - & $20(33.3)$ \\
\hline Unfavorable & - & $40(66.7)$ \\
\hline
\end{tabular}

${ }^{a}$ Data are presented as the median (25 and 75th centiles-quartiles); ${ }^{b}$ mean \pm standard deviation. AML, acute myeloid leukemia; WBC, white blood cell; PB, peripheral blood; BM, bone marrow; TIM-3, T-cell immunoglobulin and mucin domain 3; FAB, French-American-British.

software (MedCalc Software bvba). Data are presented as the mean \pm standard deviation. Unpaired Student's t-test was used to compare differences between two groups. Skewed numerical data are presented as the median and interquartile range, and the Mann-Whitney $U$ test was used to compare differences between two groups, while the Kruskal Wallis test followed by the Conover post hoc test, Bonferrroni-adjusted (critical P-value <0.005), was used to compare differences between multiple groups. Categorical variables are presented as number and percentage, and differences were compared using the Fisher's exact test. Correlations were assessed using the Spearman's rank correlation coefficient. Receiver operating characteristic (ROC) curve analysis was performed to determine the diagnostic value of TIM-3. Area under the curve (AUC) values between 0.50-0.69 represent diagnostic tests with low accuracy, while values between 0.7-0.9 represent moderate accuracy and values $>0.9$ represent high accuracy (20). The Kaplan-Meier method and log-rank test were performed to determine the prognostic value of TIM-3. $\mathrm{P}<0.05$ was considered to indicate a statistically significant difference.

\section{Results}

Clinical characteristics of patients with AML. Demographic data and baseline characteristics of the 60 patients with
AML are presented in Table I. Of the 60 patients, 8 (13.3\%) presented with EMD, including 6 men (75.0\%) and 2 women (25.0\%), and the extramedullary sites of the disease were the skin (leukemia cutis) in 3 cases and the central nervous system (CNS) in 5 cases ( 3 cases with only spinal fluid involvement and 2 cases with isolated CNS chloroma). The majority of patients with EMD (5 cases) were classified as the M4 subtype, while 2 cases were the M1 subtype and 1 case was the M0 subtype. All cases exhibited unfavourable cytogenetics.

TIM-3 expression in patients with AML and healthy individuals. TIM-3 expression was significantly upregulated in patients with AML compared with that in the healthy individuals, with median relative expression levels of 2.99 (range, 1.45-5.65) and 1.07 (0.84-1.12), respectively $(\mathrm{P}<0.001$; Fig. 1). In addition, ROC curve analysis was performed to determine the optimum cut-off value of TIM-3 expression for discrimination between patients with AML and healthy individuals. The results demonstrated a moderate diagnostic value, with an AUC value of 0.840 and optimum cut-off level $>1.197$. The diagnostic sensitivity and specificity were 81.7 and $100.0 \%$, respectively (Fig. 2).

TIM-3 expression and EMD in patients with AML. Patients with EMD exhibited significantly lower median TIM-3 


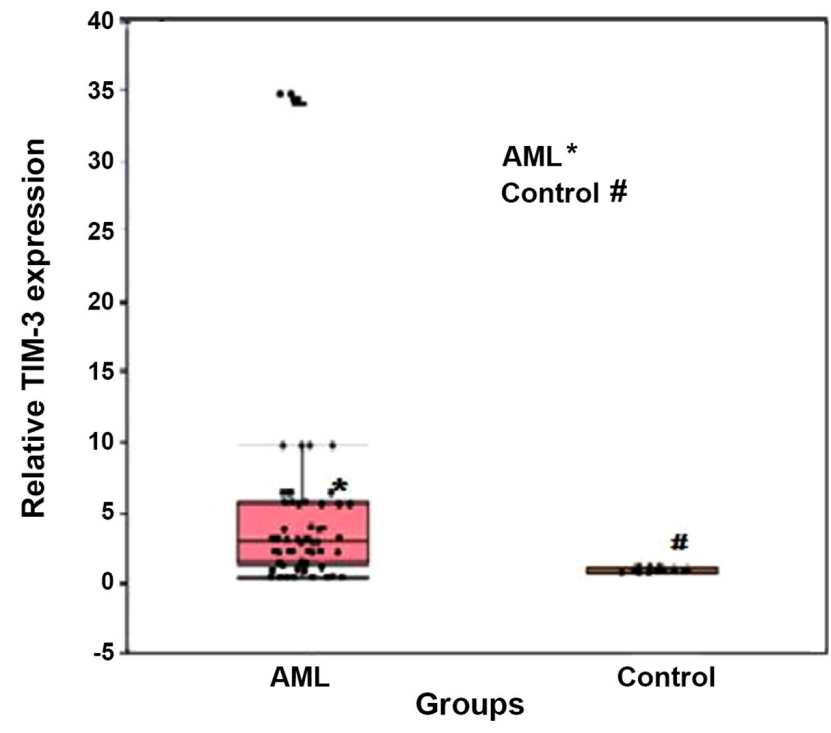

Figure 1. Box plot illustrating TIM-3 fold expression levels in both AML and control groups. Boxes represent the interquartile range. The lines inside the box represent the median. Whiskers represent minimum and maximum values. Dots represent individual observations. TIM-3, T-cell immunoglobulin and mucin domain 3; AML, acute myeloid leukemia.

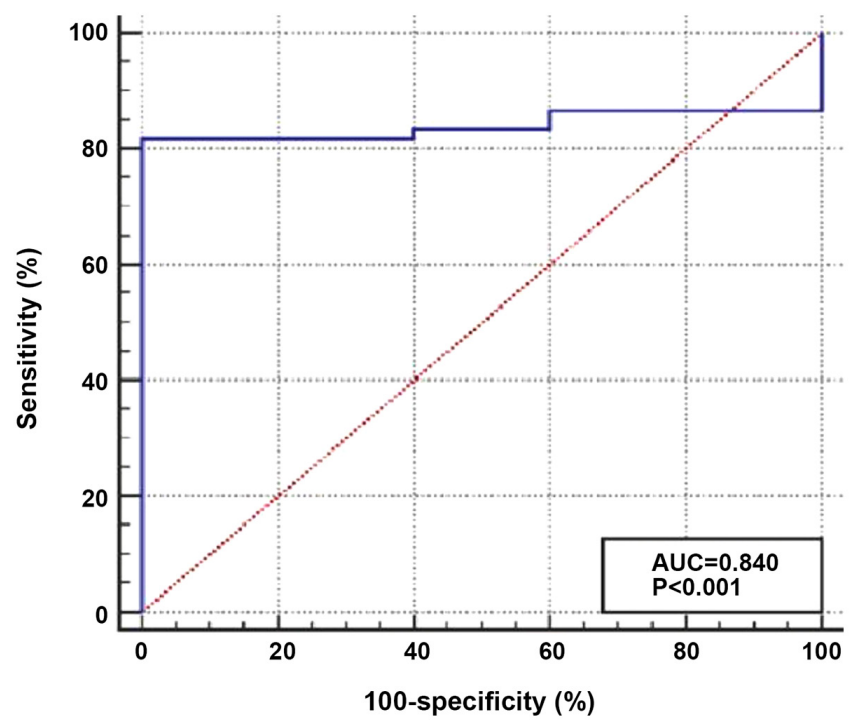

Figure 2. Receiver-operating characteristic curve for evaluating the diagnostic value of T-cell immunoglobulin and mucin domain 3 expression in acute myeloid leukemia shows a moderate diagnostic value, with an AUC value of 0.840 and an optimum cut-off level of $>1.197$. The diagnostic sensitivity and specificity were 81.7 and $100.0 \%$, respectively. AUC, area under the curve.

expression levels than those without EMD, and their median expression levels were 1.3 and 3.1 , respectively $(\mathrm{P}<0.001$; Fig. 3). ROC curve analysis was performed to determine the optimum cut-off value of TIM-3 expression for the prediction of AML cases with EMD (AUC, 0.853; optimum cut-off level, $\leq 2.2$ ). The diagnostic sensitivity and specificity were 100.0 and $69.2 \%$, respectively (Fig. 4).

Association between TIM-3 expression and the clinicopathological characteristics of patients with AML. TIM-3 expression was significantly associated with hemoglobin $(\mathrm{r}=-0.450 ; \mathrm{P}<0.001)$,

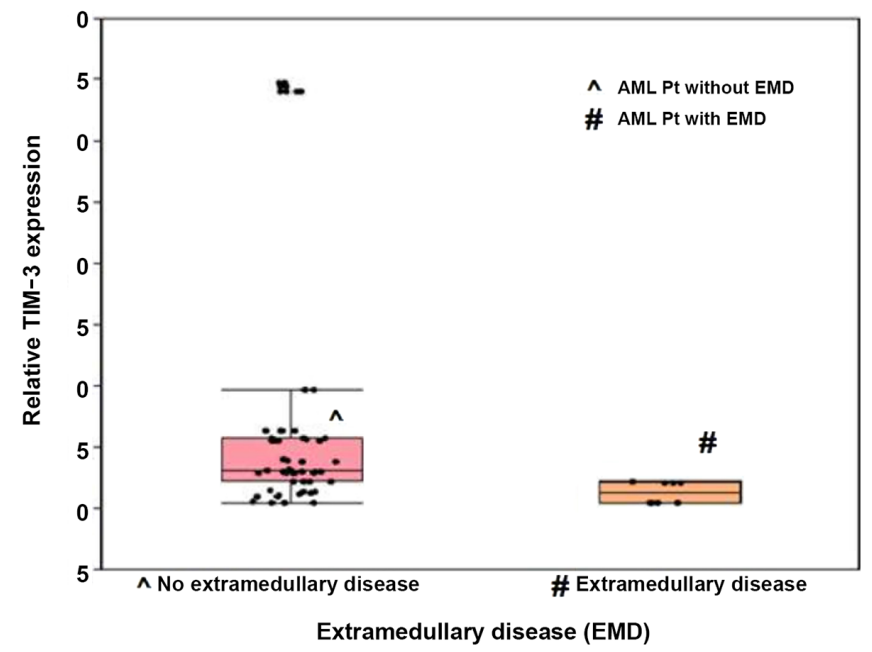

Figure 3. Box plot illustrating TIM-3 fold expression level in cases of acute myeloid leukemia with and without extramedullary disease. Boxes represent the interquartile range. The lines inside the box represent the median. Whiskers represent minimum and maximum values. Dots represent individual observations. TIM-3, T-cell immunoglobulin and mucin domain 3.

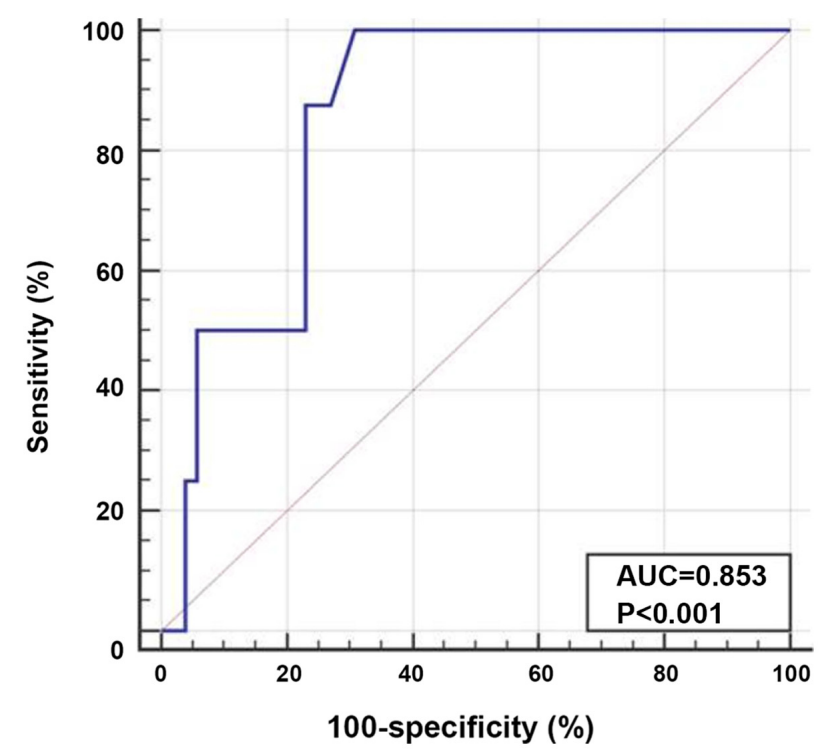

Figure 4. Receiver-operating characteristic curve for discrimination acute myeloid leukemia patients with or without extramedullary disease using T-cell immunoglobulin and mucin domain 3 expression. The AUC was 0.853 , with an optimum cut-off level of $\leq 2.2$. The diagnostic sensitivity and specificity were 100.0 and $69.2 \%$, respectively.

peripheral blood blast $(\mathrm{r}=0.324 ; \mathrm{P}=0.01)$ and $\mathrm{BM}$ blasts $(\mathrm{r}=0.300 ; \mathrm{P}=0.02)$ counts in patients with AML. However, no statistically significant associations were observed between TIM-3 expression and age, white blood cell and platelet counts (Table II).

In addition, TIM-3 expression significantly varied amongst the FAB subtypes $(\mathrm{P}<0.001)$, whereby expression was significantly higher in patients with the M1 subtype (median, 34.2; IQR, 34.00-34.59) and M4 subtype (median, 3; IQR, 2.94-6.36), compared with the other subtypes $(\mathrm{P}<0.005$; Fig. 5).

TIM-3 expression was significantly upregulated in patients with $\mathrm{CD}_{34}{ }^{+}, \mathrm{CD}_{13}{ }^{+}$and HLA-DR ${ }^{+} \mathrm{BM}$ blasts compared with 
Table II. Correlation between TIM-3 expression and variable clinicopathological parameters.

TIM-3 expression

\begin{tabular}{lcc}
\cline { 2 - 3 } Variable & Spearman $\rho$ & P-value \\
\hline Age & 0.223 & 0.087 \\
TLC & 0.121 & 0.357 \\
Hemoglobin & -0.450 & $<0.001^{\mathrm{a}}$ \\
Platelets & -0.015 & 0.912 \\
PB blasts & 0.324 & $0.012^{\mathrm{b}}$ \\
BM blasts & 0.300 & $0.020^{\mathrm{b}}$ \\
Absolute neutrophil count & 0.071 & 0.589 \\
Absolute lymphocytic count & 0.063 & 0.635
\end{tabular}

${ }^{\mathrm{a}} \mathrm{P}<0.01 ; \quad{ }^{\mathrm{b}} \mathrm{P}<0.05$. TIM-3, T-cell immunoglobulin and mucin domain 3; TLC, total leucocytic count; PB, peripheral blood; $\mathrm{BM}$, bone marrow.

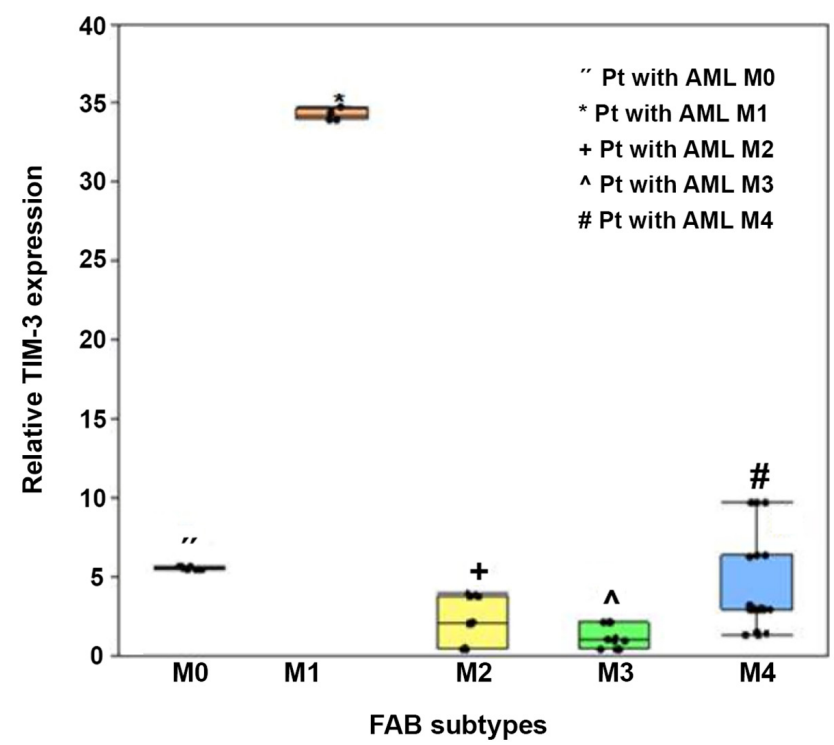

Figure 5. Box plot illustrating the association between TIM-3 expression and FAB subtypes of acute myeloid leukemia. Boxes represent the interquartile range. The lines inside the box represent the median. Whiskers represent minimum and maximum values. Dots represent individual observations. TIM-3, T-cell immunoglobulin and mucin domain 3; FAB, French-American-British.

those with $\mathrm{CD}^{-} 4^{-} \mathrm{CD}^{-}{ }^{-}$and HLA-DR ${ }^{-} \mathrm{BM}$ blasts $(\mathrm{P}=0.031$, $\mathrm{P}=0.010$ and $\mathrm{P}=0.020$, respectively; Fig. 6). Patients with unfavourable prognosis (intermediate and poor risk cytogenetics) exhibited almost comparable expression levels of TIM-3 to patients with favourable prognosis (favourable risk cytogenetics), with no statistically significant differences between the two groups ( $\mathrm{P}=0.447$; Table III).

TIM-3 expression and clinical outcomes in patients with $A M L$. To assess the impact of TIM-3 expression on the clinical outcomes of patients with AML, regarding their responsiveness to induction chemotherapy and overall 1-year survival during the first year, patients were assigned to two groups, the low TIM-3 expression group (24 patients) and the high TIM-3 expression group (36 patients), based on their median TIM-3 expression level. By the end of the induction cycle of treatment, on Day 28 (D28), the study cohorts were compared regarding their TIM-3 expression levels and treatment outcomes. A total of 12 patients (20\%) in the high TIM-3 expression group died due to pulmonary fungal infection, septicemia and bleeding complications. However, high TIM-3 expression was not significantly associated with a higher mortality risk by the end of the induction chemotherapy $(\mathrm{P}<0.09)$. The 48 survivors $(80 \%)$ underwent re-evaluation of their disease response to chemotherapy, and were further categorized into 36 responders (75\%), including 12 patients (33\%) with high TIM-3 expression and 24 patients (66\%) with low TIM-3 expression. Conversely, the 12 non-responders (20\%) with refractory disease were all categorized into the high TIM-3 expression group. Thus, all cases of AML with low TIM-3 expression were responders to induction chemotherapy, and of the patients with high TIM-3 expression, only 12 of them were responders to induction chemotherapy (Table IV). Comparison of responders vs. non-responders in terms of TIM-3 expression demonstrated that the responders had significantly lower TIM-3 expression levels compared with the non-responders ( $\mathrm{P}=0.004$; Table IV). Patients were followed up for 12 months, and of the 60 patients recruited, only 12 patients were alive at the end of the follow-up period, 8 of who had low TIM- 3 expression and 4 with high TIM-3 expression. Kaplan-Meier survival analysis demonstrated that patients with high TIM-3 expression exhibited a significantly lower overall 1-year survival rate compared with those with low TIM-3 expression ( $\mathrm{P}=0.001$; Fig. 7).

\section{Discussion}

AML is the most common type of acute leukemia in adults (21). Despite recent advancements in the therapeutic landscape of AML, patient prognosis remains poor (22). Thus, an improved understanding of the immune checkpoints and immune response to AML is imperative for appropriate development and application of novel immunotherapies (23). TIM-3 is a key immune checkpoint in tumor immune suppression; however, its role in AML remains unclear (24). Thus, the present study investigated TIM-3 expression in patients with AML and assessed its clinical significance as a potential prognostic tool for adult AML. The results demonstrated that TIM-3 expression was significantly upregulated in patients with AML compared with that in the control group. This result is consistent with previous findings, which have demonstrated that TIM-3 expression is upregulated in bone marrow samples of patients with AML via RT-qPCR analysis (25) and flow cytometric analysis of peripheral blood $\mathrm{T}$ cells or bone marrow blasts $(13,26)$. The results of the present study also demonstrated that TIM-3 expression exhibited moderate diagnostic value in the AML cohort. Research on the tumor microenvironment suggest that aberrant overexpression of TIM-3 in patients with AML may be attributed to the upregulated TIM-3 expression on the T-cell surface, mediating T-cell exhaustion in response to the continuous stimulation of malignant cell antigens, thus resulting in a weakened tumor immune 

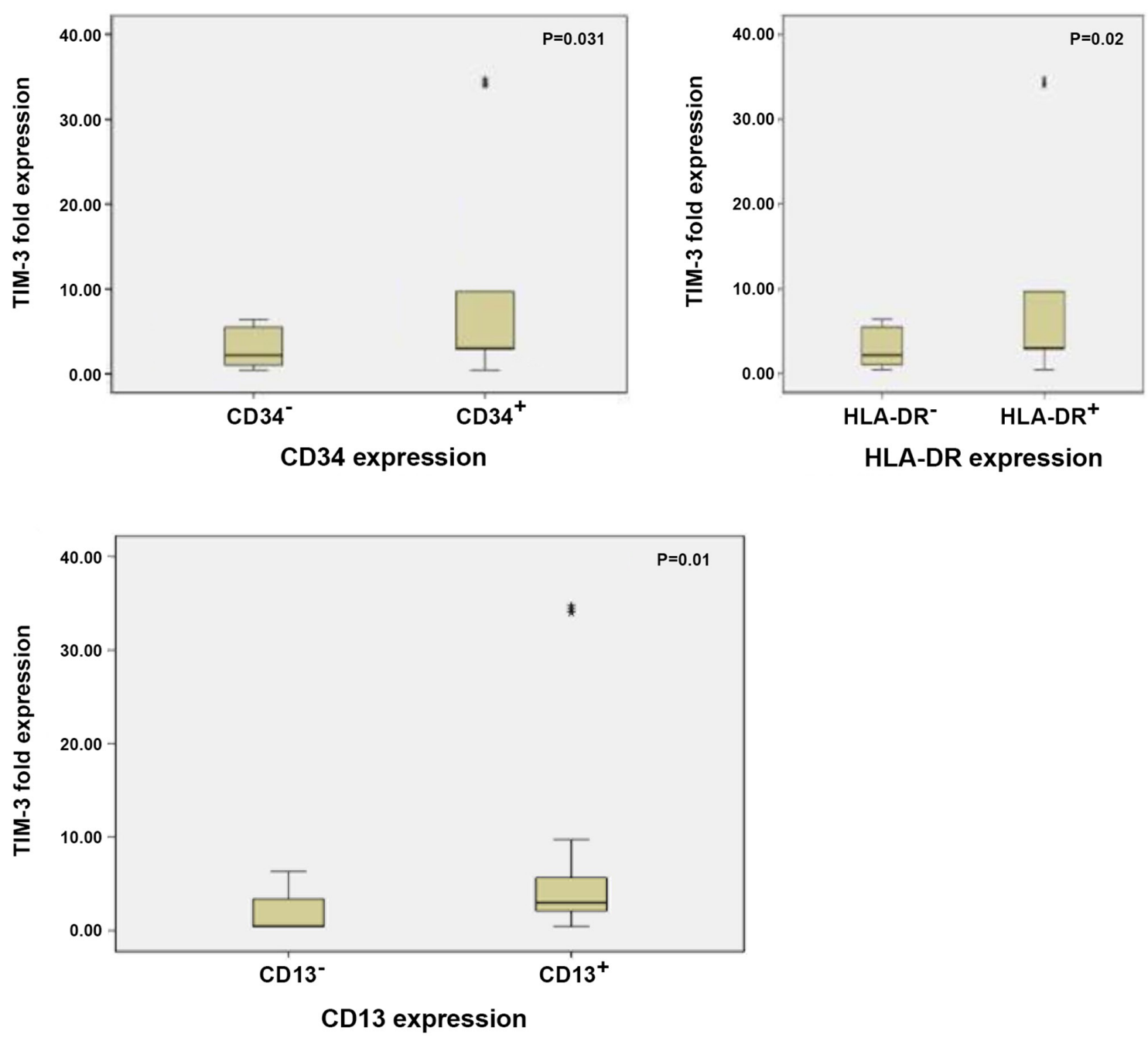

Figure 6. Box plot illustrating the TIM-3 expression level in patients with AML and bone marrow blasts positive for CD34, CD13 and HLADR, and in patients with AML and bone marrow blasts negative for these markers. Boxes represent the interquartile range. The lines inside the box represent the median. Whiskers represent minimum and maximum values. Dots represent individual observations. TIM-3, T-cell immunoglobulin and mucin domain 3; AML, acute myeloid leukemia; HLA-DR, human leukocyte antigen-DR isotype.

response (27). In addition, high TIM-3 expression on AML blasts may also explain its high level in studied AML cohorts (28). Focusing on the association between TIM-3 expression and patient characteristics, the present study demonstrated a positive correlation between TIM-3 expression and bone marrow and peripheral blast count. This result was explained in the study by Silva et al (29), which analyzed the total expression and surface presence of the TIM-3 receptor in primary human AML blasts and healthy primary human leukocytes isolated from human blood. It was reported that primary AML cells generate more TIM-3 protein compared with healthy leukocytes, including cell surface protein expression. In addition, only $30 \%$ of TIM-3 molecules are externalized in primary healthy leukocytes, while almost all TIM-3 protein is present on the cell surface of primary AML cells, facilitating its ability to mediate ligand-induced activation of the mammalian target of rapamycin pathway (29).

The results of the present study demonstrated that TIM-3 expression was significantly upregulated in patients with the M1 subtype compared with that in patients with the other FAB subtypes. Consistently, Darwish et al (25) reported that TIM-3 expression is upregulated in M1 and M4 patient groups compared with the other FAB subtypes. However, another study demonstrated that TIM-3 expression is upregulated in the M4 subtype compared with the other FAB subtypes (13).

The present study aimed to investigate the association between TIM-3 expression and different prognostic parameters in patients with AML. Cytogenetics analysis is one of the most powerful independent prognostic factors in AML; however, comparisons between TIM-3 expression in different cytogenetic risk categories in the present study failed to prove any association between cytogenetic risk groups and high TIM-3 expression. Conversely, Xu et al (26) confirmed a significant association between TIM-3 expression and inv(16), which is a favorable cytogenetic abnormality, while Li et al (13) reported that high TIM-3 expression on CD8 ${ }^{+}$ $\mathrm{T}$ cells in patients with AML is significantly associated with unfavorable cytogenetic abnormalities. The present study detected significantly higher TIM-3 expression in patients with positive expression of surface antigens CD34, CD13 and 
Table III. Association between TIM-3 expression and different cytogenetic risk groups .

Cytogenetics

\begin{tabular}{|c|c|c|c|c|c|}
\hline \multirow[b]{2}{*}{ Variable } & \multicolumn{2}{|c|}{ Favorable $(n=20)$} & \multicolumn{2}{|c|}{ Intermediate or poor $(n=40)$} & \multirow[b]{2}{*}{ P-value } \\
\hline & Median & Interquartile range & Median & Interquartile range & \\
\hline TIM-1 & 3.0 & $1.0-4.0$ & 2.9 & $2.1-5.7$ & 0.447 \\
\hline
\end{tabular}

TIM-3, T-cell immunoglobulin and mucin domain 3.

Table IV. Correlation between TIM-3 expression and post-induction chemotherapy outcome.

\begin{tabular}{lcc}
\hline Different D28 treatment outcome & Patients, n (\%) & Median TIM-3 expression \\
\hline Survival on D28 & & 2.59 \\
Alive & $48(80.0)$ & 3.05 \\
Dead & $12(20.0)$ & 2.08 \\
Response to induction chemotherapy & & 5.5 \\
Responder & $36(75.0)$ & 0.09 \\
Resistant & $12(25.0)$ & 004 \\
\hline
\end{tabular}

TIM-3, T-cell immunoglobulin and mucin domain 3. D28, day 28 at the end of induction chemotherapy cycle.

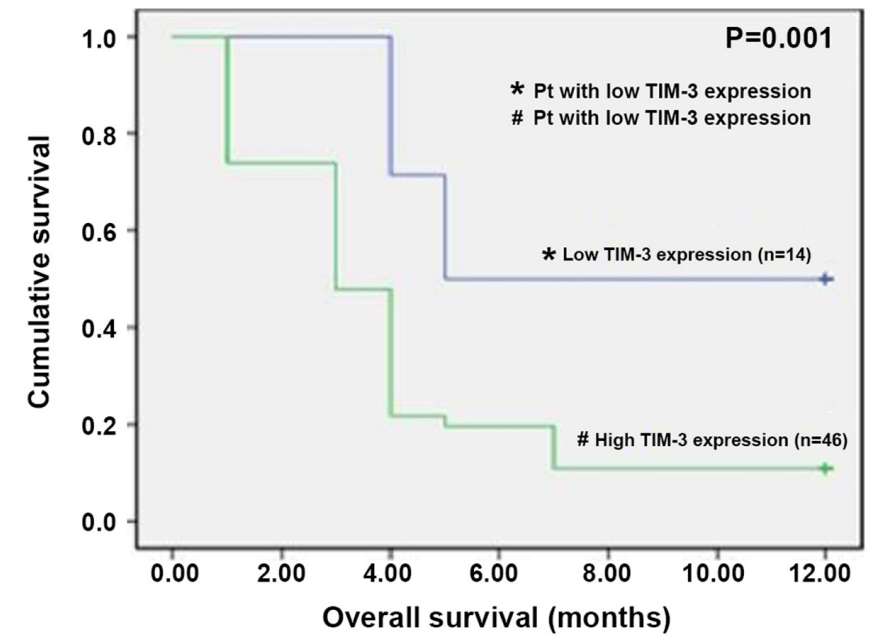

Figure 7. Kaplan-Meier survival curve demonstrating that patients with acute myeloid leukemia and a high TIM-3 expression level has a significantly lower overall survival rate than those with a low TIM-3 expression level $(\mathrm{P}=0.001)$. T-cell immunoglobulin mucin domain 3 .

HLA-DR, which are considered predictors of adverse outcome in AML $(30,31)$. These results are consistent with those in the study by $\mathrm{Xu}$ et al (26), which detected significantly positive correlations between TIM-3 expression and CD34 and CD13 in patients with AML. Several studies have reported that patients with AML and EMD have a worse prognosis due to a poor response to chemotherapy and a high relapse rate (32). To the best of our knowledge, the present study was the first to investigate the association between TIM-3 expression and
EMD in AML. Notably, the results demonstrated that TIM-3 expression was significantly downregulated in patients with EMD compared with that in those without EMD. In addition, TIM-3 expression exhibited moderate value in the prediction of AML cases with EMD. The AML cohort included 36 patients who exhibited high TIM-3 expression, while the remaining 24 patients had low TIM-3 expression. The impact of TIM-3 expression on the patient response to the induction chemotherapy protocol demonstrated that patients with low TIM-3 expression achieved a higher rate of CR compared with that of patients with high TIM-3 expression. Refractory cases exhibited significantly higher TIM-3 expression compared with the responders. These findings oppose those recorded in the study by $\mathrm{Xu}$ et al (26), which reported that patients with high TIM-3 expression achieved a significantly high $\mathrm{CR}$ rate due to the acceleration of leukemic cell apoptosis by ligand-dependent TIM-3 activation, which triggers tumor necrosis factors and induces growth factors. However, there is contradicting data to this explanation, reported in a study by Kikushige and Miyamoto (33), which demonstrated that Gal-9 produced by leukemic stem cells binds and stimulates TIM-3 expression on AML cells, including leukemia stem cells, thus supporting their survival and leukemia progression. Regarding the impact of TIM-3 expression on the overall 1-year survival of the patients assessed in the present study, the results demonstrated that patients with high TIM-3 expression had a significantly lower overall 1-year survival rate than compared with those with low TIM-3 expression. These results are consistent with those reported by Darwish et al (25), where it was demonstrated that patients with AML and low TIM-3 expression exhibit a longer 
overall survival time compared with those with high TIM-3 expression.

The present study is not without limitations. First, only 60 patients were included in the study cohort, and the cytogenetic and molecular profiles were not complete for all patients. Thus, further studies are required to confirm the expression of the immune checkpoint gene TIM-3 in a larger sample size, including full chromosomal and molecular studies. Secondly, the present study failed to investigate TIM-3 expression following chemotherapy, which would explore the correlation between the follow-up TIM-3 expression level and remission status.

In conclusion, the results of the present study demonstrated that TIM-3 expression was significantly upregulated in patients with AML. In addition, high TIM-3 expression was associated with a poor response to chemotherapy and a lower overall survival rate, suggesting that TIM-3 may act as a biomarker for a poor prognosis in AML. However, further studies are required to confirm the use of TIM-3 as a potential therapeutic target for AML.

\section{Acknowledgements}

Not applicable.

\section{Funding}

No funding was received.

\section{Availability of data and materials}

All data mentioned in this paper are available from the corresponding author and will be provided upon request.

\section{Authors' contributions}

NAN wrote the manuscript. AMK, SMR and NSE critically edited the manuscript. NAN, AMK, SMR and NSE helped to design the study, perform the research and analyze the data. NAN, SMR, NSE and AMK confirm the authenticity of all the raw data. All authors have read and approved the final manuscript.

\section{Ethics approval and consent to participate}

The study was approved by the Ethical Committee of Research, Faculty of Medicine, Ain Shams University (Cairo, Egypt) and was conducted in accordance with the Declaration of Helsinki. Consent was obtained from all individuals included in this study for their participation.

\section{Patient consent for publication}

Consent was obtained from all individuals included in this study to publish their scientific data in an aggregated anonymized manner.

\section{Competing interests}

The authors declare that they have no competing interests.

\section{References}

1. Döhner H, Estey E, Grimwade D, Amadori S, Appelbaum FR, Büchner T, Dombret H, Ebert BL, Fenaux P, Larson RA, et al: Diagnosis and management of AML in adults: 2017 ELN recommendations from an international expert panel. Blood 129: 424-47, 2017.

2. Shallis RM, Wang R, Davidoff A, Ma X and Zeidan AM: Epidemiology of acute myeloid leukemia: Recent progress and enduring challenges. Blood Rev 36: 70-87, 2019.

3. Mohammadiasl J, Khosravi A, Shahjahani M, Azizidoost S and Saki N: Molecular and cellular aspects of extramedullary manifestations of acute myeloid leukemia. J Cancer Metastasis Treat 2: 44-50, 2016

4. Wang ES: Treating acute myeloid leukemia in older adults. Hematol Am Soc Hematol Educ Program 2014: 14-20, 2014.

5. Stahl M, Lu BY, Kim TK and Zeidan AM: Novel therapies for acute myeloid leukemia: Are we finally breaking the deadlock? Target Oncol 12: 413-447, 2017.

6. Lamble AJ and Lind EF: Targeting the immune microenvironment in acute myeloid leukemia: A focus on t cell immunity. Front Oncol 8: 213, 2018.

7. Pardoll DM: The blockade of immune checkpoints in cancer immunotherapy. Nat Rev Cancer 12: 252-264, 2012.

8. Yamamoto T, Price DA, Casazza JP, Ferrari G, Nason M, Chattopadhyay PK, Roederer M, Gostick E, Katsikis PD, Douek DC, et al: Surface expression patterns of negative regulatory molecules identify determinants of virus-specific $\mathrm{CD} 8^{+}$ T-cell exhaustion in HIV infection. Blood 117: 4805-4815, 2011.

9. Anderson AC, Joller N and Kuchroo VK: Lag-3, TIM-3, and TIGIT: Co-inhibitory receptors with specialized functions in immune regulation. Immunity 44: 989-1004, 2016.

10. Zhu C, Anderson AC, Schubart A, Xiong H, Imitola J, Khoury SJ, Zheng XX, Strom TB and Kuchroo VK: The TIM-3 ligand galectin-9 negatively regulates $\mathrm{T}$ helper type 1 immunity. Nat Immunol 6: 1245-1252, 2005.

11. Anderson AC: Tim-3, a negative regulator of anti-tumor immunity. Curr Opin Immunol 24: 213-216, 2012.

12. Kikushige $\mathrm{Y}$, Shima T, Takayanagi S, Urata S, Miyamoto T, Iwasaki H, Takenaka K, Teshima T, Tanaka T, Inagaki Y and Akashi K: TIM-3 is a promising target to selectively kill acute myeloid leukemia stem cells. Cell Stem Cell 7: 708-7017, 2010.

13. Li C, Chen X, Yu X, Zhu Y, Ma C, Xia R, Ma J, Gu C, Ye L and Wu D: TIM-3 is highly expressed in T cells in acute myeloid leukemia and associated with clinicopathological prognostic stratification. Int J Clin Exp Pathol 7: 6880-6888, 2014.

14. Arber DA, Orazi A, Hasserjian R, Thiele J, Borowitz MJ, Le Beau MM, Bloomfield CD, Cazzola M and Vardiman JW: The 2016 revision to the World Health Organization classification of myeloid neoplasms and acute leukemia. Blood 127: 2391-2405, 2016.

15. Nunez R: Flow cytometry: Principles and instrumentation. Curr Issues Mol Biol 3: 39-45, 2001.

16. Fröhling S, Skelin S, Liebisch C, Scholl C, Schlenk RF, Döhner H and Döhner K; Acute Myeloid Leukemia Study Group, Ulm: Comparison of cytogenetic and molecular cytogenetic detection of chromosome abnormalities in 240 consecutive adult patients with acute myeloid leukemia. J Clin Oncol 20: 2480-2485, 2002.

17. O'Donnell MR, Abboud CN, Altman J, Appelbaum FR, Arber DA, Attar E, Borate U, Coutre SE, Damon LE, Goorha S, et al: NCCN clinical practice guidelines acute myeloid leukemia. J Natl Compr Canc Netw 10: 984-1021, 2012.

18. National Comprehensive Cancer Network: Acute myeloid leukemia (Version 2. 2018). Available at: http://www.nccn. org/professionals/physician_gls/pdf/aml.

19. Livak KJ and Schmittgen TD: Analysis of relative gene expression data using real-time quantitative PCR and the 2(-Delta Delta C(T)) method. Methods 25: 402-408, 2001.

20. Wians FH: Clinical laboratory tests: Which, why, and what do the results mean? Laboratory Med 40: 105-113, 2009.

21. Song X, Peng Y, Wang X, Chen Y, Jin L, Yang T, Qian M, Ni W, Tong $\mathrm{X}$ and Lan J: Incidence, survival, and risk factors for adults with acute myeloid leukemia not otherwise specified and acute myeloid leukemia with recurrent genetic abnormalities: Analysis of the surveillance, epidemiology, and end results (SEER) database, 2001-2013. Acta Haematol 139: 115-127, 2018.

22. Prokocimer M, Molchadsky A and Rotter V: Dysfunctional diversity of $\mathrm{p} 53$ proteins in adult acute myeloid leukemia: Projections on diagnostic work up and therapy. Blood 130: 699-712, 2017. 
23. Radwan SM, Elleboudy NS, Nabih NA and Kamal AM: The immune checkpoints cytotoxic T Lymphocyte Antigen-4 and Lymphocyte activation gene-3 expression is up-regulated in acute myeloid leukemia. HLA 96: 3-12, 2020.

24. Sakuishi K, Apetoh L, Sullivan JM, Blazar BR, Kuchroo VK and Anderson AC: Targeting TIM-3 and PD-1 pathways to reverse $\mathrm{T}$ cell exhaustion and restore anti-tumor immunity. J Exp Med 207: 2187-2194, 2010

25. Darwish NHE, Sudha T, Godugu K, Elbaz O, Abdelghaffar HA, Hassan EE and Mousa SA: Acute myeloid leukemia stem cell markers in prognosis and targeted therapy: Potential impact of BMI-1, TIM-3 and CLL-1. Oncotarget 7: 57811-57820, 2016

26. Xu L, Xu J, Ma S, Li X, Zhu M, Chen S, Han Y, Tang X, Fu Z, Qiu H, et al: High TIM-3 expression chemotherapy sensitivity on AML blasts could enhance chemotherapy sensitivity. Oncotarget 8: 102088-102096, 2017.

27. Jan M, Chao MP, Cha AC, Alizadeh AA, Gentles AJ, Weissman IL and Majeti R: Prospective separation of normal and leukemic stem cells based on differential expression of TIM3, a human acute myeloid leukemia stem cell marker. Proc Natl Acad Sci USA 108: 5009-5014, 2011.

28. Tan J, Huang S, Huang J, Yu Z, Chen Y, Lu Y, Li Y and Chen S: Increasing Tim $-3^{+} \mathrm{CD} 244^{+}$, Tim $-3^{+} \mathrm{CD} 57^{+}$, and $\mathrm{Tim}-3^{+} \mathrm{PD}-1^{+}$ $\mathrm{T}$ cells in patients with acute myeloid leukemia. Asia Pac J Clin Oncol 16: 137-141, 2020.
29. Silva IG, Gibbs BF, Bardelli M, Varani L and Sumbayev VV: Differential expression and biochemical activity of the immune receptor TIM-3 in healthy and malignant human myeloid cells. Oncotarget 6: 33823-33833, 2015.

30. Radwan SM, Hamdy NM, Hegab HM and El-Mesallamy HO Beclin-1 and hypoxia-inducible factor- $1 \alpha$ genes expression: Potential biomarkers in acute leukemia patients. Cancer Biomark 16: 619-26, 2016.

31. Sinha K: Prognostic significance of flowcytometric immunophenotype in acute leukemias: Insights gained at a tertiary care hospital. J Dental Med Sci 18: 55-58, 2019.

32. Jiang L, Yu G, Meng W, Wang Z, Meng F and Ma W: Overexpression of amyloid precursor protein in acute myeloid leukemia enhances extramedullary infiltration by MMP-2 . Tumor Biol 34: 629-636, 2013.

33. Kikushige Y and Miyamoto T: TIM-3 as a novel therapeutic target for eradicating acute myelogenous leukemia stem cells. Int J Hematol 98: 627-633, 2013.

This work is licensed under a Creative Commons Attribution-NonCommercial-NoDerivatives 4.0 International (CC BY-NC-ND 4.0) License. 